TAIWANESE JOURNAL OF MATHEMATICS

Vol. 14, No. 3A, pp. 781-793, June 2010

This paper is available online at http://www.tjm.nsysu.edu.tw/

\title{
ASYMPTOTIC PERTURBATION OF PALINDROMIC EIGENVALUE PROBLEMS
}

\author{
Tie-Xiang Li, Eric King-wah Chu* and Chern-Shuh Wang
}

\begin{abstract}
We investigate the perturbation of the palindromic eigenvalue problem for the matrix quadratic $P(\lambda) \equiv \lambda^{2} A_{1}^{T}+\lambda A_{0}+A_{1}$, with $A_{0}, A_{1} \in \mathbb{C}^{n \times n}$ and $A_{0}^{T}=A_{0}$. The perturbation of eigenvalues and eigenvectors, in terms of palindromic matrix polynomials and palindromic linearizations, are discussed using Sun's implicit function approach.
\end{abstract}

\section{INTRODUCTION}

Consider the matrix quadratic

$$
P(\lambda) \equiv \lambda^{2} A_{1}^{T}+\lambda A_{0}+A_{1}
$$

where $A_{0}, A_{1} \in \mathbb{C}^{n \times n}$ and $A_{0}^{T}=A_{0}$, and the corresponding palindromic quadratic eigenvalue problem

$$
P(\lambda) x=0, \quad x \neq 0 .
$$

In this paper, we shall consider only regular matrix polynomials $P(\lambda)$, in the sense that $\operatorname{det} P(\lambda) \not \equiv 0$.

From the transpose of (1), a palindromic eigenvalue problem possesses a spectrum $\sigma(P)$ containing both $\lambda$ and its reciprocal $\lambda^{-1}$ (with 0 and $\infty$ considered to be reciprocal to each other). When $\lambda \neq-1$, the eigenvalue problem of the original matrix polynomial $P(\lambda)$ has a palindromic linearization $[5,8,14]$ of the form $\lambda Z \pm Z^{T}$. (We can transform $\lambda Z-Z^{T}$ to the form $\nu(-Z)+(-Z)^{T}$ with $\nu=-\lambda$. Similarly, $\lambda^{2} A_{1}^{T}+\lambda A_{0}+A_{1}$ and $\nu^{2} A_{1}^{T}-\nu A_{0}+A_{1}$ define equivalent palindromic eigenvalue problems).

Received October 18, 2008, accepted July 14, 2009.

2000 Mathematics Subject Classification: 15A18, 15A22, 65F15.

Key words and phrases: Eigenvalue, Eigenvector, Implicit function theorem, Matrix polynomial, Palindromic eigenvalue problem, Palindromic linearization, Perturbation.

*Corresponding author. 
A great foundation for the solution of palindromic eigenvalue problems has been laid by Hilliges, Mackey, Mehl and Mehrmann [11, 14, 15]. Alternative approaches in tackling the problem with structure-preserving doubling algorithms and generalized Patel/Analdi method can be found in [5, 12]. There has been much recent interest in quadratic eigenvalue problems [20]. An important example of palindromic eigenvalue problems can be found in the vibration analysis of fast trains; see [11, 13] for general introductions and [10] for details. For general perturbation of eigenvalues for polynomial eigenvalue problems, see [4, 19]. On results for general matrix polynomials, see the masterpiece [8].

This paper is organized as follows. The perturbation result for a simple eigenvalue and its corresponding eigenvector is obtained by differentiation in Section 2. Sun's implicit function approach [18] is then applied to palindromic linearizations, general matrix quadratics and palindromic eigenvalue problems in Section 3, to obtain perturbation results for (simple) eigenvalues and the corresponding eigenvectors/deflating subspaces. An illustrative example is given in Section 4 and the paper is concluded in Section 5.

Some related results for perturbation of arbitrary size, in terms of Bauer-Fike type theorems, can be found in [6]. Although we have only considered the Tpaldindromic problems here, related H-palindromic, anti-palindromic and odd/even problems can be treated similarly.

\section{Perturbation by Differentiation}

Without establishing the differentiability or the existence of asymptotic expansions (which can be achieved using the implicit function approach in the next section), perturbation results can be obtained by simple differentiation. See [1] for more details on this approach.

For some fixed $z \neq 0$, consider the palindromic eigenvalue problem

$$
P(\lambda, \rho) x(\rho)=0, \quad P(\lambda, \rho) \equiv \lambda(\rho)^{2} A_{1}^{T}(\rho)+\lambda(\rho) A_{0}(\rho)+A_{1}(\rho)
$$

with the scaling $z^{T} x(\rho)-1=0$, where $\rho$ is the perturbation parameter, $A_{0}(0)=A_{0}$ and $A_{1}(0)=A_{1}$. We shall use the subscripts $(\cdot)_{\rho}$ and $(\cdot)_{\lambda}$ to denote the corresponding partial derivatives. For a simple eigenvalue $\lambda$, differentiation produces, at $\rho=0$ :

$$
\lambda_{\rho}=-\frac{y^{T} P_{\rho} x}{y^{T} P_{\lambda} x}=-\frac{y^{T} P_{\rho} x}{y^{T}\left(2 \lambda A_{1}^{T}+A_{0}\right) x}
$$

and

$$
P x_{\rho}=-\left(\lambda_{\rho} P_{\lambda}+P_{\rho}\right) x, \quad z^{T} x_{\rho}=0 .
$$


Choosing $z=y(0)$ (the left-eigenvector corresponding to $\lambda(0)$ ), we have, at $\rho=0$ :

$$
x_{\rho}=-P^{\dagger}\left(\lambda_{\rho} P_{\lambda}+P_{\rho}\right) x
$$

where $P^{\dagger}$ denotes the Penrose generalized inverse [9].

The usual conclusion can be drawn - the right-eigenvector $x$ will be rotated through a big angle, even for a small perturbation, when $\left\|P^{\dagger}\right\|$ is large, i.e., when the separation between $\lambda$ and other eigenvalues is fine. This happens, of course, when the assumption of simplicity for the eigenvalue is near collapsing.

Note that for palindromic eigenvalues problems, $\lambda= \pm 1$ may be multiple and non-differentiable, and a more sophisticated approach, like the one in [3], is required. This comment is also applicable to Sun's approach in the next Section.

\section{Sun's Implicit Function ApProAch}

In this Section, we shall apply Sun's approach $[7,18]$ where the implicit function theorem is applied. Asymptotic perturbation series for the eigenvalues and the corresponding deflating subspaces are obtained.

\subsection{Palindromic pemcils}

From [16], we have the following anti-triangular canonical form:

Theorem 1. Let $Z-\lambda Z^{T}$ be a regular $n \times n$ palindromic linearization. There exists a unitary $U \in \mathbb{C}^{n \times n}$ such that $U^{T} Z U=\left(m_{i j}\right)$ with $m_{i j}=0(i+j \leq n+1)$ (i.e., $U^{T} Z U$ is anti-triangular, with zero elements in the upper left corner).

The palindromic eigenvalues are:

$$
\frac{m_{1 n}}{m_{n 1}}, \frac{m_{2, n-1}}{m_{n-1,2}}, \cdots, \frac{m_{i, n-i+1}}{m_{n-i+1, i}}, \cdots, \frac{m_{n-i+1, i}}{m_{i, n-i+1}}, \cdots, \frac{m_{n-1,2}}{m_{2, n-1}}, \frac{m_{n 1}}{m_{1 n}} .
$$

Using the anti-triangular form in Theorem 1, Sun's approach [7] can be applied to obtain the power series of eigenvalues and deflating subspaces. Without loss of generality, we shall use an upper-triangular form in this section (with the help of the order-reversing permutation matrix $\mathcal{P}_{r} \equiv\left[e_{r}, e_{r-1}, \cdots, e_{1}\right] \in \mathbb{R}^{r \times r}$ with $e_{i}$ being the $i$ th column of the $r$-dimensional identity matrix $I_{r}$, so that Sun's approach can be followed faithfully).

Using $\mathcal{P}_{n}$, the anti-triangular form (in Theorem 1) is turned into a upper triangular form and is organized to reflect the symmetry of the eigenvalue pairs $\left\{\lambda_{j}, \lambda_{j}^{-1}\right\}$ :

$$
\mathcal{P}_{n} U^{T}\left(Z-\lambda Z^{T}\right) U
$$




$$
\begin{aligned}
& =\left[\begin{array}{cc}
\Lambda_{\alpha_{1}} & * \\
0 & \Lambda_{\alpha 2}
\end{array}\right]-\lambda\left[\begin{array}{cc}
\Lambda_{\beta_{1}} & * \\
0 & \Lambda_{\beta 2}
\end{array}\right] \\
& =\left[\begin{array}{ccc}
\Lambda_{\alpha 1} & & * \\
& \Lambda_{\alpha 0} & \\
0 & & \Lambda_{\alpha,-1}
\end{array}\right]-\lambda\left[\begin{array}{ccc}
\Lambda_{\beta 1} & & * \\
& \Lambda_{\beta 0} & \\
0 & & \Lambda_{\beta,-1}
\end{array}\right] .
\end{aligned}
$$

With $2 p<n,\left(\Lambda_{\alpha 1}, \Lambda_{\beta 1}\right)$ contains the $p$ eigenvalues whose perturbation we are interested in, $\left(\Lambda_{\alpha,-1}, \Lambda_{\beta,-1}\right)$ their reciprocals, $\left(\Lambda_{\alpha 0}, \Lambda_{\beta 0}\right)$ the rest of the spectrum, and $\left(\Lambda_{\alpha 2}, \Lambda_{\beta 2}\right)$ the complement of those in $\left(\Lambda_{\alpha 1}, \Lambda_{\beta 1}\right)$. Consequently, we have

$$
\Lambda_{\beta,-1}=\mathcal{P}_{p} \Lambda_{\alpha 1} \mathcal{P}_{p}, \quad \Lambda_{\beta, 1}=\mathcal{P}_{p} \Lambda_{\alpha,-1} \mathcal{P}_{p}, \quad \Lambda_{\beta 0}=\mathcal{P}_{2 n-p} \Lambda_{\alpha 0} \mathcal{P}_{2 n-p}
$$

and $\left\{U_{-1}, U_{1}\right\}$ spanning the deflating subspaces of $Z-\lambda Z^{T}$ corresponding to $\left(\Lambda_{\alpha 1}, \Lambda_{\beta 1}\right)$. With similar organization of the deflating subspaces, we have

$$
U \equiv\left[U_{1}, U_{0}, U_{-1}\right]=\left[U_{1}, U_{2}\right]=\left[U_{-2}, U_{-1}\right], U_{2} \equiv\left[U_{0}, U_{-1}\right], U_{-2} \equiv\left[U_{1}, U_{0}\right]
$$

and

$$
U \mathcal{P}_{n}=\left[U_{-1} \mathcal{P}_{p}, U_{0} \mathcal{P}_{n-2 p}, U_{1} \mathcal{P}_{p}\right]=\left[U_{-1} \mathcal{P}_{p}, U_{-2} \mathcal{P}_{n-p}\right]=\left[U_{2} \mathcal{P}_{n-p}, U_{1} \mathcal{P}_{p}\right]
$$

Here $U_{1}$ (corresponding to $\left(\Lambda_{\alpha 1}, \Lambda_{\beta 1}\right)$ ) and $U_{-1}$ (corresponding to the "reciprocal" pencil $\left.\left(\Lambda_{\alpha,-1}, \Lambda_{\beta,-1}\right)\right)$ are respectively the first and last $p$ columns of $U$.

Assume that $\left(\Lambda_{\alpha 1}, \Lambda_{\beta 1}\right)$ and $\left(\Lambda_{\alpha 2}, \Lambda_{\beta 2}\right)$ have nonitersecting spectra. (This excludes the possibility of $\lambda= \pm 1$ appearing in both $\left(\Lambda_{\alpha,-1}, \Lambda_{\beta,-1}\right)$ and $\left(\Lambda_{\alpha 1}, \Lambda_{\beta 1}\right)$. All these unimodular eigenvalues have to be grouped together inside $\left(\Lambda_{\alpha 1}, \Lambda_{\beta 1}\right)$, with the necessary adjustment of the $U$ s.) We assume that $Z(\rho)$ is analytic with respect to the perturbation parameter $\rho$. (Frequently, the dependence on $\rho$ is not written explicitly to avoid messy expressions.) We then have

$$
\mathcal{M} \equiv \mathcal{P}_{n} U^{T} Z(\rho) U=\left[\begin{array}{ll}
\mathcal{M}_{11} & \mathcal{M}_{12} \\
\mathcal{M}_{21} & \mathcal{M}_{22}
\end{array}\right] \equiv\left[\begin{array}{cc}
\mathcal{P}_{p} U_{1}^{T} Z U_{-1} & \mathcal{P}_{p} U_{1}^{T} Z U_{-2} \\
\mathcal{P}_{n-p} U_{2}^{T} Z U_{-1} & \mathcal{P}_{n-p} U_{2}^{T} Z U_{-2}
\end{array}\right]
$$

and

$$
\mathcal{L} \equiv \mathcal{P}_{n} U^{T} Z(\rho)^{T} U=\left[\begin{array}{cc}
\mathcal{L}_{11} & \mathcal{L}_{12} \\
\mathcal{L}_{21} & \mathcal{L}_{22}
\end{array}\right] \equiv\left[\begin{array}{cc}
\mathcal{P}_{p} U_{1}^{T} Z^{T} U_{-1} & \mathcal{P}_{p} U_{1}^{T} Z^{T} U_{-2} \\
\mathcal{P}_{n-p} U_{2}^{T} Z^{T} U_{-1} & \mathcal{P}_{n-p} U_{2}^{T} Z^{T} U_{-2}
\end{array}\right] .
$$

From the (2,1)-block of

$$
\left[\begin{array}{cc}
I_{p} & 0 \\
-\Psi & I_{n-p}
\end{array}\right](\mathcal{M}-\lambda \mathcal{L})\left[\begin{array}{cc}
I_{p} & 0 \\
\Phi & I_{n-p}
\end{array}\right]
$$


with $\Psi(\rho)$ and $\Phi(\rho)$ being functions of $\rho$, we construct

$$
\begin{aligned}
& {\left[\begin{array}{c}
F(\Phi, \Psi, \rho) \\
G(\Phi, \Psi, \rho)
\end{array}\right] } \\
= & {\left[\begin{array}{c}
-\Psi \mathcal{P}_{p} U_{1}^{T} Z U_{-1}-\Psi \mathcal{P}_{p} U_{1}^{T} Z U_{-2} \Phi+\mathcal{P}_{n-p} U_{2}^{T} Z U_{-1}+\mathcal{P}_{n-p} U_{2}^{T} Z U_{-2} \Phi \\
-\Psi \mathcal{P}_{p} U_{1}^{T} Z^{T} U_{-1}-\Psi \mathcal{P}_{p} U_{1}^{T} Z^{T} U_{-2} \Phi+\mathcal{P}_{n-p} U_{2}^{T} Z^{T} U_{-1}+\mathcal{P}_{n-p} U_{2}^{T} Z^{T} U_{-2} \Phi
\end{array}\right] . }
\end{aligned}
$$

At $\rho=0$, we have $\Phi=0=\Psi$ and $F(0,0,0)=0=G(0,0,0)$. The appropriate implicit function theorem can then be applied to $\left[F^{T}, G^{T}\right]^{T}$. Differentiation of $F$ and $G$ respect to $\Psi$ and $\Phi$ at $\rho=0$ yields (after stacking columns and apply Kronecker products)

$$
\left[\begin{array}{c}
F \\
G
\end{array}\right]_{(\Psi, \Phi)}=\left[\begin{array}{cc}
-\Lambda_{\alpha 1}^{T} \otimes I_{n-p} & I_{p} \otimes \Lambda_{\alpha 2} \\
-\Lambda_{\beta 1}^{T} \otimes I_{n-p} & I_{p} \otimes \Lambda_{\beta 2}
\end{array}\right] .
$$

It is easy to see that the above operator is invertible when the spectra of $\left(\Lambda_{\alpha 1}, \Lambda_{\beta 1}\right)$ and $\left(\Lambda_{\alpha 2}, \Lambda_{\beta 2}\right)$ do not intersect [2] (and it will be ill-conditioned when the separation of the spectra is small). Consequently, we have proved that the power series exist for $\Phi(\rho)$ and $\Psi(\rho)$ within some small neighbourhood of $\rho=0$, and

$$
\Phi(\rho)=\Phi_{\rho}(0) \rho+\cdots, \quad \Psi(\rho)=\Psi_{\rho}(0) \rho+\cdots .
$$

Furthermore, the perturbed deflation subspaces [7] are

$$
\begin{aligned}
& \left\{\operatorname{span}\left(\bar{U} \mathcal{P}_{n}\left[\begin{array}{c}
I_{p} \\
\Psi(p)
\end{array}\right]\right), \operatorname{span}\left(U\left[\begin{array}{c}
I_{p} \\
\Phi(p)
\end{array}\right]\right)\right\} \\
= & \left\{\operatorname{span}\left(\bar{U}_{1} \mathcal{P}_{n}+\bar{U}_{2} \mathcal{P}_{n-p} \Psi(p)\right), \operatorname{span}\left(U_{-1}+U_{-2} \Phi(p)\right)\right\} .
\end{aligned}
$$

Differentiation of

$$
\mathcal{M}_{11} \equiv \mathcal{P}_{p} U_{1}^{T} Z U_{-1}, \quad \mathcal{L}_{11} \equiv \mathcal{P}_{p} U_{1}^{T} Z^{T} U_{-1}
$$

also yields, at $\rho=0$ :

$$
\frac{\partial \mathcal{M}_{11}}{\partial \rho}=\mathcal{P}_{p} U_{1}^{T} Z_{\rho} U_{-1}, \quad \frac{\partial \mathcal{L}_{11}}{\partial \rho}=\mathcal{P}_{p} U_{1}^{T} Z_{\rho}^{T} U_{-1} .
$$

Applying the trace operator to the above derivatives will produce the derivatives of the averages of multiple eigenvalues [3]. Alternatively, subgradients can be applied for such analysis. Note that the multiple eigenvalues $\lambda= \pm 1$ are of particular interest in the study of palindromic eigenvalue problems and their perturbation analysis can be performed using the above formulae $[5,14,15,16]$. 
When $p=1$ and $\left(\Lambda_{\alpha 1}, \Lambda_{\beta 1}\right)=\left(\lambda_{\alpha 1}, \lambda_{\beta 1}\right)$ represents the finite eigenvalue $\lambda_{1}=\lambda_{\alpha 1} / \lambda_{\beta 1}$, the above derivatives translate to

$$
\frac{\partial \lambda_{1}}{\partial \rho}=\frac{\frac{\partial \lambda_{\alpha 1}}{\partial \rho} \lambda_{\beta 1}-\frac{\partial \lambda_{\beta 1}}{\partial \rho} \lambda_{\alpha 1}}{\lambda_{\beta 1}^{2}}=\frac{\frac{\partial \lambda_{\alpha 1}}{\partial \rho}}{\lambda_{\beta 1}}-\frac{\lambda_{1} \frac{\partial \lambda_{\beta 1}}{\partial \rho}}{\lambda_{\beta 1}}=\frac{y_{1}^{T} Z_{\rho} x_{1}-\lambda_{1} y_{1}^{T} Z_{\rho}^{T} x_{1}}{y_{1}^{T} Z^{T} x_{1}},
$$

producing a result analogous to (2).

Lastly, differentiating $F$ and $G$ with respect to $\rho$ at $\rho=0$ produces

$$
\begin{aligned}
& F_{\rho}=\Lambda_{\alpha 2} \Phi_{\rho}-\Psi_{\rho} \Lambda_{\alpha 1}+\mathcal{P}_{n-p} U_{2}^{T} Z_{\rho} U_{-1}=0 \\
& G_{\rho}=\Lambda_{\beta 2} \Phi_{\rho}-\Psi_{\rho} \Lambda_{\beta 1}+\mathcal{P}_{n-p} U_{2}^{T} Z_{\rho}^{T} U_{-1}=0
\end{aligned}
$$

The derivatives $\Phi_{\rho}(0)$ and $\Psi_{\rho}(0)$ (required in (??) when calculating the perturbed deflating subspaces) can then be retrieved from the above equations, when $\left(\Lambda_{\alpha 1}, \Lambda_{\beta 1}\right)$ and $\left(\Lambda_{\alpha 2}, \Lambda_{\beta 2}\right)$ have nonintersecting spectra [2].

\subsection{General matrix quadratics}

We now apply Sun's approach [7] to the general matrix quadratic

$$
Q_{2}(\lambda)=\lambda^{2} M+\lambda D+K
$$

similar to the development in the previous subsection. Assume the $n \times 2 n$ matrices $X=\left[x_{j}\right]$ and $Y=\left[y_{j}\right]$ contain, respectively, the right- and left-eigenvectors $x_{j}$ and $y_{j}$ corresponding to $\lambda_{j}=\alpha_{j} / \beta_{j}$. For the companion linearization

$$
\mathcal{L}(\lambda) \equiv\left[\begin{array}{rr}
0 & I \\
-K & -D
\end{array}\right]-\lambda\left[\begin{array}{ll}
I & \\
& M
\end{array}\right]
$$

it is easy to check that the right- and left-eigenvectors corresponding to $\lambda_{j}=\alpha_{j} / \beta_{j}$ are respectively

$$
\left[\begin{array}{c}
\beta_{j} x_{j} \\
\alpha_{j} x_{j}
\end{array}\right],\left[\beta_{j} y_{j}^{T} D+\alpha_{j} y_{j}^{T} M, \beta_{j} y_{j}^{T}\right]^{T}
$$

Notice that by using $\left(\alpha_{j}, \beta_{j}\right)$ to represent $\lambda_{j}$, we have avoided the difficulties involving infinite eigenvalues. We can then scale the eigenvectors to satisfy the biorthogonality equations

$$
\begin{array}{r}
\Lambda_{\alpha} Y^{T} M X \Lambda_{\beta}+\Lambda_{\beta} Y^{T} M X \Lambda_{\alpha}+\Lambda_{\beta} Y^{T} D X \Lambda_{\beta}=\Lambda_{\beta}, \\
\Lambda_{\alpha} Y^{T} M X \Lambda_{\alpha}-\Lambda_{\beta} Y^{T} K X \Lambda_{\beta}=\Lambda_{\alpha} .
\end{array}
$$


Here $\Lambda_{\alpha}$ and $\Lambda_{\beta}$ are block-diagonal matrices, with blocks being the usual identity or Jordan matrices for the generalized eigenvalue sub-problems.

Assume further that $X, Y, \Lambda_{\alpha}$ and $\Lambda_{\beta}$ are organized as follows:

$$
X=\left[X_{1}, X_{2}\right], Y=\left[Y_{1}, Y_{2}\right] ; \Lambda_{\alpha}=\operatorname{diag}\left\{\Lambda_{\alpha 1}, \Lambda_{\alpha 2}\right\}, \Lambda_{\beta}=\operatorname{diag}\left\{\Lambda_{\beta 1}, \Lambda_{\beta 2}\right\}
$$

where the spectra of $\left(\Lambda_{\alpha 1}, \Lambda_{\beta 1}\right)$ and $\left(\Lambda_{\alpha 2}, \Lambda_{\beta 2}\right)$ do not intersect.

We assume the matrices $M, D$ and $K$ are differentiable with respect to the perturbation parameter $\rho$. We first transform the linearization in (4) by pre-(post)multiplying with its left-(right-)eigenvectors:

$$
\mathcal{M} \equiv\left[\begin{array}{ll}
\mathcal{M}_{11} & \mathcal{M}_{12} \\
\mathcal{M}_{21} & \mathcal{M}_{22}
\end{array}\right] \equiv\left[\Lambda_{\beta} Y^{T} D+\Lambda_{\alpha} Y^{T} M, \Lambda_{\beta} Y^{T}\right]\left[\begin{array}{rr}
0 & I \\
-K & -D
\end{array}\right]\left[\begin{array}{l}
X \Lambda_{\beta} \\
X \Lambda_{\alpha}
\end{array}\right]
$$

and

$$
\mathcal{L} \equiv\left[\begin{array}{cc}
\mathcal{L}_{11} & \mathcal{L}_{12} \\
\mathcal{L}_{21} & \mathcal{L}_{22}
\end{array}\right] \equiv\left[\Lambda_{\beta} Y^{T} D+\Lambda_{\alpha} Y^{T} M, \Lambda_{\beta} Y^{T}\right]\left[\begin{array}{ll}
I & \\
& M
\end{array}\right]\left[\begin{array}{l}
X \Lambda_{\beta} \\
X \Lambda_{\alpha}
\end{array}\right] .
$$

From the $(2,1)$-block of

$$
\left[\begin{array}{cc}
I_{p} & 0 \\
-\Psi & I_{n-p}
\end{array}\right](\mathcal{M}-\lambda \mathcal{L})\left[\begin{array}{cc}
I_{p} & 0 \\
\Phi & I_{n-p}
\end{array}\right]
$$

with $\Psi(\rho)$ and $\Phi(\rho)$ being functions of $\rho$, we construct

$$
\begin{aligned}
F(\Phi, \Psi, \rho) \equiv & -\Psi\left(\Lambda_{\alpha 1} Y_{1}^{T} M X_{1} \Lambda_{\alpha 1}-\Lambda_{\beta 1} Y_{1}^{T} K X_{1} \Lambda_{\beta 1}\right) \\
& -\Psi\left(\Lambda_{\alpha 1} Y_{1}^{T} M X_{2} \Lambda_{\alpha 2}-\Lambda_{\beta 1} Y_{1}^{T} K X_{2} \Lambda_{\beta 2}\right) \Phi \\
& +\left(\Lambda_{\alpha 2} Y_{2}^{T} M X_{1} \Lambda_{\alpha 1}-\Lambda_{\beta 2} Y_{2}^{T} K X_{1} \Lambda_{\beta 1}\right) \\
& +\left(\Lambda_{\alpha 2} Y_{2}^{T} M X_{2} \Lambda_{\alpha 2}-\Lambda_{\beta 2} Y_{2}^{T} K X_{2} \Lambda_{\beta 2}\right) \Phi \\
G(\Phi, \Psi, \rho) \equiv & -\Psi\left(\Lambda_{\beta 1} Y_{1}^{T} D X_{1} \Lambda_{\beta 1}+\Lambda_{\alpha 1} Y_{1}^{T} M X_{1} \Lambda_{\beta 1}+\Lambda_{\beta 1} Y_{1}^{T} M X_{1} \Lambda_{\alpha 1}\right) \\
& -\Psi\left(\Lambda_{\beta 1} Y_{1}^{T} D X_{2} \Lambda_{\beta 2}+\Lambda_{\alpha 1} Y_{1}^{T} M X_{2} \Lambda_{\beta 2}+\Lambda_{\beta 1} Y_{1}^{T} M X_{2} \Lambda_{\alpha 2}\right) \Phi \\
& +\left(\Lambda_{\beta 2} Y_{2}^{T} D X_{1} \Lambda_{\beta 1}+\Lambda_{\alpha 2} Y_{2}^{T} M X_{1} \Lambda_{\beta 1}+\Lambda_{\beta 2} Y_{2}^{T} M X_{1} \Lambda_{\alpha 1}\right) \\
& +\left(\Lambda_{\beta 2} Y_{2}^{T} D X_{2} \Lambda_{\beta 2}+\Lambda_{\alpha 2} Y_{2}^{T} M X_{2} \Lambda_{\beta 2}+\Lambda_{\beta 2} Y_{2}^{T} M X_{2} \Lambda_{\alpha 2}\right) \Phi .
\end{aligned}
$$

At $\rho=0$, we have $\Phi=0=\Psi$ and $F(0,0,0)=0=G(0,0,0)$. The complex implicit function theorem can then be applied to $\left(F^{T}, G^{T}\right)^{T}$. Differentiation of $F$ and $G$ respect to $\Psi$ and $\Phi$ at $\rho=0$ yields (after stacking columns and apply Kronecker products)

$$
\left[\begin{array}{c}
F \\
G
\end{array}\right]_{(\Psi, \Phi)}=\left[\begin{array}{cc}
-\Lambda_{\alpha 1}^{T} \otimes I_{p} & I_{n-p} \otimes \Lambda_{\alpha 2} \\
-\Lambda_{\beta 1}^{T} \otimes I_{p} & I_{n-p} \otimes \Lambda_{\beta 2}
\end{array}\right]
$$


It is easy to see that the above operator is invertible when the spectra of $\left(\Lambda_{\alpha 1}, \Lambda_{\beta 1}\right)$ and $\left(\Lambda_{\alpha 2}, \Lambda_{\beta 2}\right)$ do not intersect [2] (and it will be ill-conditioned when the separation of the spectra is small). Consequently, we have proved that the power series exists for $\Phi(\rho)$ and $\Psi(\rho)$ within some small neighbourhood of $\rho=0$, and

$$
\Phi(\rho)=\Phi_{\rho}(0) \rho+\cdots, \quad \Psi(\rho)=\Psi_{\rho}(0) \rho+\cdots .
$$

Furthermore, the perturbed deflation subspaces [7] of the companion linearization equal

$$
\left\{\operatorname{span}\left(\tilde{Y}^{-T}\left[\begin{array}{c}
I_{p} \\
\Psi(p)
\end{array}\right]\right), \operatorname{span}\left(\tilde{X}\left[\begin{array}{c}
I_{p} \\
\Phi(p)
\end{array}\right]\right)\right\}
$$

with

$$
\tilde{Y}=\left[\Lambda_{\beta} Y^{T} D+\Lambda_{\alpha} Y^{T} M, \Lambda_{\beta} Y^{T}\right]^{T}, \quad \tilde{X}=\left[\begin{array}{c}
X \Lambda_{\beta} \\
X \Lambda_{\alpha}
\end{array}\right]
$$

Differentiation of

$$
\begin{aligned}
\mathcal{M}_{11} & \equiv \Lambda_{\alpha 1} Y_{1}^{T} M X_{1} \Lambda_{\alpha 1}-\Lambda_{\beta 1} Y_{1}^{T} K X_{1} \Lambda_{\beta 1} \\
\mathcal{L}_{11} & \equiv \Lambda_{\beta 1} Y_{1}^{T} D X_{1} \Lambda_{\beta 1}+\Lambda_{\alpha 1} Y_{1}^{T} M X_{1} \Lambda_{\beta 1}+\Lambda_{\beta 1} Y_{1}^{T} M X_{1} \Lambda_{\alpha 1}
\end{aligned}
$$

also yields, at $\rho=0$ :

$$
\begin{aligned}
& \frac{\partial \mathcal{M}_{11}}{\partial \rho}=\Lambda_{\alpha 1} Y_{1}^{T} M_{\rho} X_{1} \Lambda_{\alpha 1}-\Lambda_{\beta 1} Y_{1}^{T} K_{\rho} X_{1} \Lambda_{\beta 1} \\
& \frac{\partial \mathcal{L}_{11}}{\partial \rho}=\Lambda_{\beta 1} Y_{1}^{T} D_{\rho} X_{1} \Lambda_{\beta 1}+\Lambda_{\alpha 1} Y_{1}^{T} M_{\rho} X_{1} \Lambda_{\beta 1}+\Lambda_{\beta 1} Y_{1}^{T} M_{\rho} X_{1} \Lambda_{\alpha 1} .
\end{aligned}
$$

Again for multiple eigenvalues (like $\lambda= \pm 1$ ), applying the trace operator to the above derivatives will produce the derivatives of the averages of multiple eigenvalues [3]; otherwise subgradients will be required.

When $p=1$ and $\left(\Lambda_{\alpha 1}, \Lambda_{\beta 1}\right)=\left(\lambda_{\alpha 1}, \lambda_{\beta 1}\right)$ represents the finite eigenvalue $\lambda_{1}=\lambda_{\alpha 1} / \lambda_{\beta 1}$, the above derivatives translate to

$$
\begin{aligned}
& \frac{\partial \lambda_{1}}{\partial \rho} \\
= & \frac{\frac{\partial \lambda_{\alpha 1}}{\partial \rho} \lambda_{\beta 1}-\frac{\partial \lambda_{\beta 1}}{\partial \rho} \lambda_{\alpha 1}}{\lambda_{\beta 1}^{2}}=\frac{\frac{\partial \lambda_{\alpha 1}}{\partial \rho}}{\lambda_{\beta 1}}-\frac{\lambda_{1} \frac{\partial \lambda_{\beta 1}}{\partial \rho}}{\lambda_{\beta 1}} \\
= & \frac{-\lambda_{\beta 1}^{2} y_{1}^{T} K_{\rho} x_{1}+\lambda_{\alpha 1}^{2} y_{1}^{T} M_{\rho} x_{1}-\lambda_{1}\left(\lambda_{\beta 1}^{2} y_{1}^{T} D_{\rho} x_{1}+2 \lambda_{\alpha 1} \lambda_{\beta 1} y_{1}^{T} M_{\rho} x_{1}\right)}{\lambda_{\beta 1}^{2} y_{1}^{T} D x_{1}+2 \lambda_{\alpha 1} \lambda_{\beta 1} y_{1}^{T} M x_{1}} \\
= & -\frac{y_{1}^{T}\left(\lambda_{1}^{2} M_{\rho}+\lambda_{1} D_{\rho}+K_{\rho}\right) x_{1}}{y_{1}^{T}\left(2 \lambda_{1} M+D\right) x_{1}},
\end{aligned}
$$


producing a formula similar to (2).

Lastly, differentiating $F$ and $G$ with respect to $\rho$ at $\rho=0$ produces

$$
\begin{aligned}
F_{\rho}= & \Lambda_{\alpha 2} \Phi_{\rho}-\Psi_{\rho} \Lambda_{\alpha 1}+\Lambda_{\alpha 2} Y_{2}^{T} M_{\rho}(0) X_{1} \Lambda_{\alpha 1}-\Lambda_{\beta 2} Y_{2}^{T} K_{\rho}(0) X_{1} \Lambda_{\beta 1}=0 \\
G_{\rho}= & \Lambda_{\beta 2} \Phi_{\rho}-\Psi_{\rho} \Lambda_{\beta 1}+\Lambda_{\alpha 2} \Lambda_{\beta 2} Y_{2}^{T} D_{\rho}(0) X_{1} \Lambda_{\beta 1} \\
& \quad+\Lambda_{\alpha 2} Y_{2}^{T} M_{\rho}(0) X_{1} \Lambda_{\beta 1}+\Lambda_{\beta 2} Y_{2}^{T} M_{\rho}(0) X_{1} \Lambda_{\alpha 1}=0
\end{aligned}
$$

The derivatives $\Phi_{\rho}(0)$ and $\Psi_{\rho}(0)$ can then be retrieved from the above equations, when $\left(\Lambda_{\alpha 1}, \Lambda_{\beta 1}\right)$ and $\left(\Lambda_{\alpha 2}, \Lambda_{\beta 2}\right)$ have nonintersecting spectra [2].

\subsection{Palindromic case}

For palindromic eigenvalue problems, the perturbation results can be obtained from those for general matrix quadratics, utilizing $M=A_{1}^{T}=K^{T}, D=A_{0}=D^{T}$ and the palindromic properties of the eigenvalues and eigenvectors. For the spectrum, we have $\left(\Lambda_{\alpha 1}, \Lambda_{\beta 1}\right)$ and $\left(\Lambda_{\alpha 2}, \Lambda_{\beta 2}\right)=\left(\Lambda_{\beta 1}, \Lambda_{\alpha 1}\right)$ representing, respectively, eigenvalues on/inside and on/outside the unit circle, and $\left[Y_{1}, Y_{2}\right]=\left[X_{2}, X_{1}\right]$.

For the simple palindromic eigenvalues $\lambda_{1}$ and $\lambda_{1}^{-1}$, (??) implies, at $\rho=0$ :

$$
\begin{array}{r}
\frac{\partial \lambda_{1}}{\partial \rho}=-\frac{y_{1}^{T}\left[\lambda_{1}^{2}\left(A_{1}\right)_{\rho}^{T}+\lambda_{1}\left(A_{0}\right)_{\rho}+\left(A_{1}\right)_{\rho}\right] x_{1}}{y_{1}^{T}\left(2 \lambda_{1} A_{1}^{T}+A_{0}\right) x_{1}}, \\
\frac{\partial\left\{\lambda_{1}^{-1}\right\}}{\partial \rho}=\frac{y_{1}^{T}\left[\left(A_{1}\right)_{\rho}^{T}+\lambda_{1}^{-1}\left(A_{0}\right)_{\rho}+\lambda_{1}^{-2}\left(A_{1}\right)_{\rho}\right] x_{1}}{y_{1}^{T}\left(2 \lambda_{1} A_{1}^{T}+A_{0}\right) x_{1}}
\end{array}
$$

from $\frac{\partial\left\{\lambda_{1}^{-1}\right\}}{\partial \rho}=-\lambda_{1}^{-2} \frac{\partial \lambda_{1}}{\partial \rho}$ or $\left(2 \lambda_{1} A_{1}+A_{0}\right) x_{1}=-\left(2 \lambda_{1}^{-1} A_{1}^{T}+A_{0}\right) x_{1}$. Interestingly, the asymptotic relative errors of the pair of reciprocal eigenvalues equal to, at $\rho=0$ :

$$
\frac{\rho}{\lambda_{1}^{ \pm 1}} \frac{\partial \lambda_{1}^{ \pm 1}}{\partial \rho}=\mp \rho \frac{y_{1}^{T}\left[\lambda_{1}\left(A_{1}\right)_{\rho}^{T}+\left(A_{0}\right)_{\rho}+\lambda_{1}^{-1}\left(A_{1}\right)_{\rho}\right] x_{1}}{y_{1}^{T}\left(2 \lambda_{1} A_{1}^{T}+A_{0}\right) x_{1}} .
$$

These are identical except of the opposite signs. Equation (8) can easily be understood through

$$
\frac{1}{\lambda+\delta \lambda}=\frac{1}{\lambda\left(1+\frac{\delta \lambda}{\lambda}\right)} \approx \frac{1}{\lambda}\left(1-\frac{\delta \lambda}{\lambda}\right) \Rightarrow \frac{(\lambda+\delta \lambda)^{-1}-\lambda^{-1}}{\lambda^{-1}} \approx-\frac{\delta \lambda}{\lambda} .
$$

After applying inequalities of norms, a condition number for both $\lambda_{1}^{ \pm 1}$ can then be produced:

$$
\kappa \equiv \frac{\left\|y_{1}\right\|_{2}\left\|x_{1}\right\|_{2}}{\left|y_{1}^{T}\left(2 \lambda_{1} A_{1}^{T}+A_{0}\right) x_{1}\right|} \sqrt{\left|\lambda_{1}\right|^{2}+1+\left|\lambda_{1}\right|^{-2}} .
$$


Table 1: Perturbation of a palindromic eigenvalue problem

\begin{tabular}{l|lll}
\hline$i$ & 1 & 2,3 & 4 \\
\hline$\lambda_{i}$ & -0.5488554937 & $0.6854143467 \pm$ & -1.8219731997 \\
& & $0.7281532623 \mathrm{i}$ & \\
$\tilde{\lambda}_{i}$ & -0.5488554724 & $0.6854143626 \pm$ & -1.8219732705 \\
& & $0.7281532473 \mathrm{i}$ & \\
$\delta \lambda_{i}$ & $2.1312649867 \mathrm{e}-08$ & $1.5930742392 \mathrm{e}-08 \pm$ & $-7.0749180514 \mathrm{e}-08$ \\
& & $1.4995688913 \mathrm{e}-08 \mathrm{i}$ & \\
$r_{i}$ & $-3.8831076873 \mathrm{e}-08$ & $-4.1288419705 \mathrm{e}-16 \pm$ & $3.8831076399 \mathrm{e}-08$ \\
& & $2.1878282362 \mathrm{e}-08 \mathrm{i}$ & \\
$\left|r_{i}\right|$ & $3.8831076873 \mathrm{e}-08$ & $2.1878282362 \mathrm{e}-08$ & $3.8831076399 \mathrm{e}-08$ \\
$r_{i}^{(e)}$ & $-3.8831078433 \mathrm{e}-08$ & $4.7865922782 \mathrm{e}-16 \pm$ & $3.8831078433 \mathrm{e}-08$ \\
$\left|r_{i}^{(e)}\right|$ & $3.8831078433 \mathrm{e}-08$ & $2.1878282332 \mathrm{e}-08 \mathrm{i}$ & \\
& & & \\
$\kappa_{i}$ & 1.2701114034 & 1.5072107111 & 1.2701114034 \\
$r_{i}^{(\kappa)}$ & $8.8772549782 \mathrm{e}-08$ & $1.0534425368 \mathrm{e}-07$ & $8.8772549782 \mathrm{e}-08$ \\
\hline
\end{tabular}

With appropriate scaling of the eigenvectors, $\kappa$ can be interpreted as proportional to the product of the norms of the left- and the right-eigenvectors, or in terms of the angle between the eigenvectors, as in other algebraic eigenvalue problems.

\section{NUMERICAL EXAMPLE}

We shall consider the following small example to illustrate the results in Section 7.3:

$$
A_{0}=\left[\begin{array}{ll}
1 & 2 \\
2 & 1
\end{array}\right], \quad A_{1}=\left[\begin{array}{cc}
1 & \omega \\
0 & 1
\end{array}\right] .
$$

The parameter $\omega$ can be used to vary the condition of the eigenvalue problem, but will be fixed to be 0.5 in the following calculations. The matrices are perturbed randomly to the magnitude of $0.5 \times 10^{-7}$, with $\delta=\left\|\left[\delta A_{1}^{T}, \delta A_{0}, \delta A_{1}\right]\right\|=$ $0.6989351449543007 \times 10^{-7}$. The eigenvalues are $\lambda_{i}(i=1, \cdots, 4)$ with $\lambda_{1}=\lambda_{4}^{-1}$ and $\lambda_{2}=\lambda_{3}^{-1}$. The numerical results are summarized in Table 1 , with $\tilde{\lambda}_{i}$ denoting the perturbed eigenvalues, $\delta \lambda_{i} \equiv \tilde{\lambda}_{i}-\lambda_{i}, r_{i} \equiv \delta \lambda_{i} / \lambda_{i}$ (the relative error in $\left.\tilde{\lambda}_{i}\right), r_{i}^{(e)}$ estimating $r_{i}$ using (8), $\kappa_{i}$ the individual condition numbers as in (9), and $\left|r_{i}^{(e)}\right| \leq r_{i}^{(\kappa)} \equiv \kappa_{i} \delta$ estimating $\left|r_{i}\right|$. All calculations were carried out using MATLAB 7.1 (R14) on a Apple MacIntosh G4 Powerbook, with eps $\approx 2.2204 \times 10^{-16}$. 
It is obvious from Table 1 that (8) provides accurate approximations to the relative errors of palindromic eigenvalues for small perturbations and the condition number $\kappa$ in (9) produces tight upper bounds for the (relative) errors. Also, the fact that the relative errors for a reciprocal pairs of eigenvalues are negative of each other is confirmed by the example.

\section{Conclusions}

Results for eigenvalues and the corresponding deflating subspaces of palindromic linearizations and (palindromic) matrix quadratics are obtained using Sun's implicit function approach. Consistent results for simple eigenvalues and the corresponding eigenvetors/deflating subspaces are obtained using simple differentiation. These results indicate, not surprisingly, that the perturbations of an eigenvalue $\lambda$ and its corresponding deflating subspace $\mathcal{S}_{\lambda}$, respectively, are proportional to the size of the perturbation and the reciprocal of the gap between $\mathcal{S}_{\lambda}$ and other deflating subspaces. Condition numbers are typically proportional the products of the norms of the leftand right-eigenvectors or deflating subspaces.

\section{REFERENCES}

1. A. L. Andrew, K.-W. E. Chu and P. Lancaster, Derivatives of eigenvalues and eigenvectors of nonlinear eigenvalue problems, SIAM J. Matrix Anal. Applic., 14 (1993), 903-926.

2. K.-W. E. Chu, The solution of the matrix equations $A X B-C X D=E$ and $(Y A-$ $D Z, Y C-B Z)=(E, F)$, Lin. Alg. Applic., 93 (1987), 93-105.

3. K.-W. E. Chu, On multiple eigenvalues of matrices depending on several parameters, SIAM J. Numer. Anal., 27 (1990), 1368-1385.

4. K.-W. E. Chu, Perturbation of eigenvalues for matrix polynomials via the Bauer-Fike theorems, SIAM J. Matrix Anal. Applic., 25 (2003), 551-573.

5. K.-W. E. Chu, T.-M. Hwang, W.-W. Lin and C.-T. Wu, Vibration of fast trains, palindromic eigenvalue problems and structure-preserving doubling algorithms, $J$. Comput. Appl. Maths., 219 (2007), 237-252. (doi: 10.1016/j.cam.2007.07.016).

6. E. K.-W. Chu, W.-W. Lin and C.-S. Wang, Perturbation results related to palindromic eigenvalue problems, Preprints and Reprints, National Centre of Theoretical Sciences, National Tsing Hua University, Taiwan, August 2007. ANZIAM J., to appear.

7. L. Elsner and J. G. Sun, Perturbation theorems for the generalized eigenvalue problem, Lin. Alg. Applic., 48 (1982), 341-357. 
8. I. Gohberg, P. Lancaster and L. Rodman, Matrix Polynomials, Academic Press, New York, 1982.

9. G. H. Golub and C. F. Van Loan, Matrix Computations, 3rd Ed., Johns Hopkins University Press, Baltimore, 1996.

10. A. Hilliges, Numerische Lösung von quadratischen Eigenwertproblemen mit Anwendungen in der Schienendynamik, Master Thesis, Technical University Berlin, Germany, July 2004.

11. A. Hilliges, C. Mehl and V. Mehrmann, On the solution of palindromic eigenvalue problems, Proceedings 4th European Congress on Computational Methods in Applied Sciences and Engineering (ECCOMAS), Jyväskylä, Finland, 2004.

12. T.-M. Huang, W.-W. Lin and J. Qian, Structured algorithms for palindromic quadratic eigenvalue problems arising in vibration of fast trains, Technical report, NCTS Preprints in Mathematics, National Tsing Hua University, Hsinchu, Taiwan, 2008-2001, 2008, SIAM J. Matrix Anal. Applic.), to appear.

13. C. F. Ipsen, Accurate eigenvalues for fast trains, SIAM News, 37 (2004).

14. D. S. Mackey, N. Mackey, C. Mehl and V. Mehrmann, Vector spaces of linearizations for matrix polynomials, SIAM J. Matrix Applic., 28 (2006), 971-1004.

15. D. S. Mackey, N. Mackey, C. Mehl and V. Mehrmann, Palindromic polynomial eigenvalue problems: good vibrations from good linearizations, SIAM J. Matrix Applic., 28 (2006), 1029-1051.

16. D. S. Mackey, N. Mackey, C. Mehl and V. Mehrmann, Numerical methods for palindromic eigenvalue problems: computing the anti-triangular Schur form, Technical Report 409, DFG Research Centre Matheon, Berlin, 2007, Numer. Lin. Alg. Appl., to appear.

17. Mathworks, MATLAB User's Guide, 2002.

18. G. W. Stewart and J. G. Sun, Matrix Perturbation Theory, Academic Press, New York, 1990.

19. F. Tisseur, Backward error and condition of polynomial eigenvalue problems, Lin. Alg. Applic., 309 (2000), 339-361.

20. F. Tisseur and K. Meerbergen, A survey of the quadratic eigenvalue problem, SIAM Rev., 43 (2001), 234-286.

Tie-Xiang Li

Department of Mathematics,

Southeast University,

Nanjing 211189,

P. R. China

E-mail: txli@seu.edu.cn 
Eric King-wah Chu

School of Mathematical Sciences,

Building 28, Monash University,

VIC 3800, Australia

E-mail: eric.chu@sci.monash.edu.au

Chern-Shuh Wang

Department of Mathematics,

National Cheng Kung University,

Tainan 701, Taiwan

E-mail: cswang@math.ncku.edu.tw 